\title{
Chemical Switching of the Magnetic Coupling in a MnPc Dimer by Means of Chemisorption and Axial Ligands
}

\author{
Barbara Brena,* Biplab Sanyal, and Heike C. Herper
}

Cite This: J. Phys. Chem. C 2020, 124, 27185-27193

Read Online

ABSTRACT: We present an $a b$ initio density functional theory study of the magnetic properties of manganese phthalocyanine dimers, where we focus on the magnetic coupling between the Mn centers and on how it is affected by external factors like chemisorption or atomic axial ligands. We have studied several different configurations for the gas phase dimers, which resulted in ferromagnetic couplings of different magnitudes. For the bare dimer we find a significant ferromagnetic coupling between the Mn centers, which decreases by about $20 \%$ when a $\mathrm{H}$ atom is adsorbed

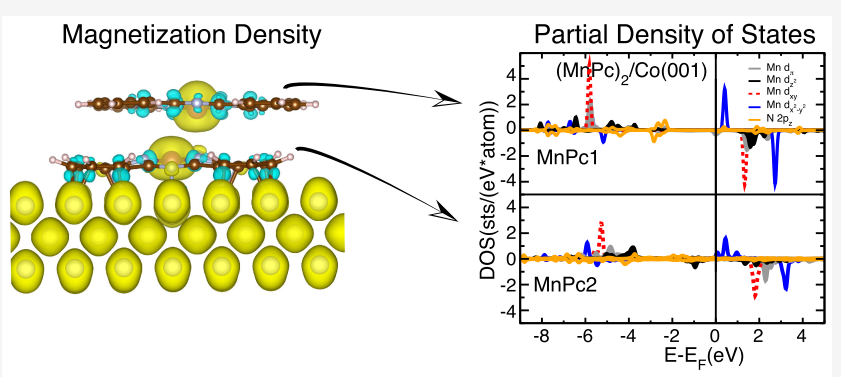
on one of the $\mathrm{Mn}$ atoms and is reduced to about $7 \%$ when a $\mathrm{Cl}$ atom is adsorbed. The magnetic coupling is almost fully quenched when the dimer, bare or with the $\mathrm{H}$ ligand, is deposited on the ferromagnetic substrate $\mathrm{Co}(001)$. Our calculations indicate that the coupling between the two $\mathrm{Mn}$ atoms principally occurs via a superexchange interaction along two possible paths within a Mn-N$\mathrm{Mn}-\mathrm{N}$ four-atom loop. When these electrons get involved in chemical bonding outside the dimer itself, an appreciable alteration of the overlap between $\mathrm{Mn}$ and $\mathrm{N}$ molecular orbitals along the loop occurs, and consequently, the magnetic interaction between the Mn centers varies. We show that this is reflected by the electronic structure of the dimer in various configurations and is also visible in the structure of the atomic loop. The chemical tuning of the magnetic coupling is highly relevant for the design of nanodevices like molecular spin valves, where the molecules need to be anchored to a support.

\section{INTRODUCTION}

A key objective of molecular electronics and spintronics (spinbased electronics) is the downscaling of the electronic components, which would bring up remarkable benefits like increase in magnetic storage density and reduction of power consumption. In this context, the possibility to use organic molecular materials of low production cost has become highly appealing. The molecules of the phthalocyanine family (Pc) have been studied quite extensively in the emerging field of organic spintronics. ${ }^{1-4}$ Among the milestones in this field are the possibility of spin-polarized injection and transport of electrons through organic semiconductors, shown by Dediu and colleagues, ${ }^{5}$ and the giant magnetoresistance observed for a single $\mathrm{H}_{2} \mathrm{Pc}^{1}$ and CoPc. ${ }^{6}$ In addition, an organic spin valve device based on immobilized layers of $\mathrm{CuPc}$ (spacer layer) and aligned $\mathrm{MnPc}$ (or NiPc) as spin-injection and spin-detection layers was fabricated and studied experimentally by Banerjee and co-workers. ${ }^{7}$ Recently, also supermolecular nano and lowdimensional structures like molecular chains and dimers have been addressed, with an emphasis on their structures ${ }^{8}$ and magnetic properties. ${ }^{9,10}$ These systems are promising novel building blocks for molecular size devices as well as model systems to understand the magnetic interactions and spin transfer mechanisms between the molecules or between the molecules and substrates. An important issue in this field is to find feasible mechanisms to manipulate the molecular spin and magnetic moments in a reproducible fashion.

In the present work, we target dimers of the MnPc molecule, which is among the most studied phthalocyanines in molecular spintronics, due to the known magnetic interactions in $\mathrm{MnPc}$ based bulk materials. ${ }^{11,12}$ Interestingly, the deposition of $\mathrm{MnPc}$ molecules on the ferromagnetic $\mathrm{Co}(001)$ surface has shown to generate a so-called spinterface, ${ }^{13,14}$ i.e., an active heterogeneous interface for spin transport. ${ }^{3}$ Beyond that, $\mathrm{MnPc}$ and FePc coadsorbed in the form of a checkerboard table on $\mathrm{Au}(111)$ were reported to be antiferromagnetically coupled through the Ruderman-Kittel-Kasuya-Yosida (RKKY) exchange interaction via the substrate electronic states. ${ }^{15} \mathrm{MnPc}$, shown in Figure 1A, consists of a single Mn center surrounded by an organic ring, representing a sort of archetype of single molecule magnet. The molecule has a spin $3 / 2$ and a $D_{2 h}$ symmetry. The magnetic properties of specific phases of $\mathrm{MnPc}$ films were described already some decades ago. Both

Received: September 16, 2020

Revised: November 10, 2020

Published: November 24, 2020

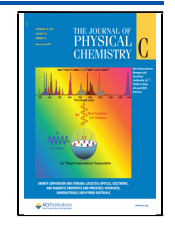




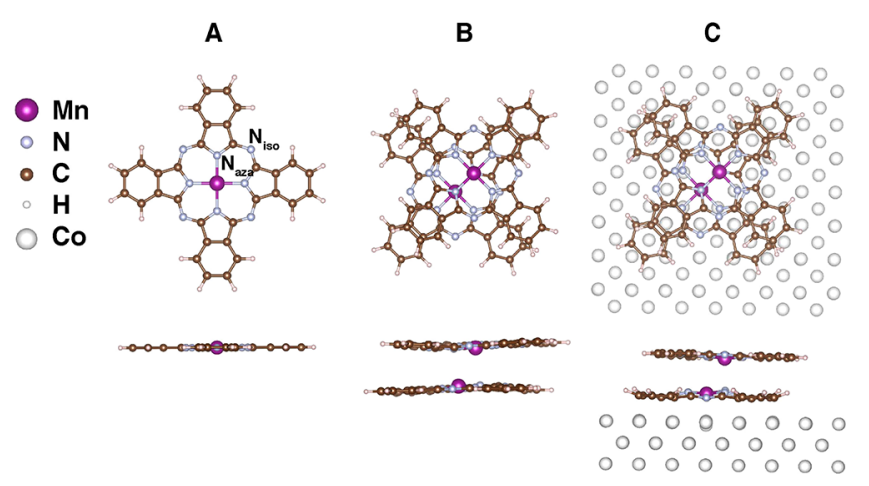

Figure 1. (A) Top view and side view of $\mathrm{MnPc}$, where isoindole $\mathrm{N}$ $\left(\mathrm{N}_{\text {iso }}\right)$ and azabridge $\mathrm{N}\left(\mathrm{N}_{\text {aza }}\right)$ type atoms are highlighted. (B) Top view and side view of the MnPc dimer in the $\alpha+$ configuration. (C) Top view and side view of the MnPc dimer in the $\alpha+$ configuration adsorbed on the $\mathrm{Co}(001)$ surface.

ferromagnetic and antiferromagnetic couplings were reported for respectively the $\beta$ and $\alpha$ phases in bulk MnPc films, ${ }^{11,12}$ showing that the magnetic interaction between the molecules is directly linked to their reciprocal arrangement. ${ }^{12}$ Because of this, it is essential to unveil how the interplay between the electronic and geometric structure shapes the magnetic properties to engineer the future molecule-scale devices.

We have first studied the magnetic properties of dimers of $\mathrm{MnPc}$ in the gas phase, considering several possible molecular configurations by means of $a b$ initio density functional theory (DFT) including dispersion interactions. We have then focused on the dimer with the configuration of lowest energy and studied its adsorption on a $\mathrm{Co}(001)$ substrate, both bare and with atomic axial ligands such as $\mathrm{H}$ and $\mathrm{Cl}$. It has been shown in previous joint theory/experiment studies how these theoretical methods well reproduce the experimental findings for the adsorbate $\mathrm{MnPc} / \mathrm{Co}(100)$ system in terms of adsorption distances and magnetic properties.,16 Previous theoretical studies have reported dimers of phthalocyanines in geometrical configurations that are derived from the various $3 \mathrm{D}$ bulk stackings, ${ }^{8}$ where the magnetic interactions are directly associated with those in the thick films. We have here analyzed the connection between the magnetic coupling and the inter- and intramolecular chemical bondings that occur in the dimer. The Mn atoms at the center of the molecules are coupled via superexchange interaction, which unrolls via a $\mathrm{N}$ atom directly bonded to one of the $\mathrm{Mn}$ atoms and situated in the face of the $\mathrm{Mn}$ of the opposing molecule, via a mechanism analogous to the one described for $\mathrm{MnPc}$ films and bulk materials. ${ }^{11,12}$ This type of superexchange path was also experimentally investigated by Chen et al. ${ }^{17}$ by means of spinflip electron tunneling spectroscopy between CoPc molecules adsorbed on a $\mathrm{Pb}(111)$ surface, where the CoPc molecules assume a reciprocal configuration analogous to the one of the dimers in our study. We observe that when the $\mathrm{Mn}$ atoms take part in intermolecular chemical bonds, the magnetic coupling is markedly reduced. This happens in all cases when the dimer is chemisorbed on a Co surface via one of the MnPc rings or when a $\mathrm{H}$ or a $\mathrm{Cl}$ atom is axially adsorbed on the $\mathrm{Mn}$ center on one side of the dimer. In these cases, the chemical bonding scheme of the $\mathrm{Mn}$ atoms is changed, as can be seen in the modifications of the inter- and intramolecular distances between the $\mathrm{Mn}$ and $\mathrm{N}$ atoms involved.

\section{THEORETICAL METHODS}

The present study is based on $a b$ initio DFT calculations using the VASP ${ }^{18}$ code with the Perdew, Burke, and Ernzerhof (PBE) exchange correlation functional ${ }^{19,20}$ and the projector augmented wave method. ${ }^{21}$ The plane wave cutoff was $400 \mathrm{eV}$, and only the $\Gamma$-point $k$-mesh was used. An effective Hubbard term $U_{\text {eff }}$ of $4 \mathrm{eV}$ was chosen to describe the Mn $3 \mathrm{~d}$ orbitals, following Dudarev's method. ${ }^{22}$ The value for the Hubbard term has been chosen according to the findings in our previous study. ${ }^{23}$ This value has been proven to properly reproduce experimental data, for example, photoemission spectra. ${ }^{23}$ The long-range dispersion forces were described with Grimme's second method (D2). ${ }^{24}$ For comparison, some tests were performed with the (D3) ${ }^{25}$ method showing no differences in the relevant properties. To describe the Co(001) substrate, we have used a three-layer fcc Co film as in our previous works with $\mathrm{FePc}$ on $\mathrm{Co}(001),{ }^{16,26}$ with a lattice constant of $3.61 \AA$. For all the calculations, from the single molecule in gas phase to the dimers adsorbed on $\mathrm{Co}(001)$, we employed a cubic super cell of size $25.53 \AA$. In this way, when the cell contains the Co slab plus the $\mathrm{MnPc}$ dimer, a vacuum layer of about 17.5 $\AA$ is obtained between the upper MnPc and the next Co slab. The lateral distance between two MnPc molecules is about 15 $\AA$. The density of states (DOS) curves were calculated by using 2000 points in the range of -30 to $10 \mathrm{eV}$.

\section{RESULTS AND DISCUSSION}

We have first analyzed different possible geometries for the $\mathrm{MnPc}$ dimer based on possible arrangements of multilayers of metal Pc molecules. We will briefly discuss these structures which are shown in Figure S1 of the Supporting Information. Starting from the $\alpha$ and $\beta$ polymorphs, we have built the corresponding models $\alpha \times$ with a tilt angle of about $63^{\circ}$, the $\alpha+$ with a tilt angle of $59^{\circ}$ (Figure $1 \mathrm{~B}$ ), and the $\beta$ type with a tilt angle of $43^{\circ}$, where the tilt angle is the angle between the line joining the two $\mathrm{Mn}$ atoms and a line orthogonal to the plain of the molecules. In the $\alpha+$ and $\beta$ configurations, the two molecules are shifted with respect to each other along an axis joining the $\mathrm{Mn}$ center to an isoindole $\mathrm{N}$ atom $\left(\mathrm{N}_{\mathrm{iso}}\right)$, while in the $\alpha$ polymorph, the reciprocal shift occurs along a line joining the $\mathrm{Mn}$ center to an aza bridge $\mathrm{N}$ atom $\left(\mathrm{N}_{\mathrm{aza}}\right)$ (see Figure 1A). The $\alpha+$ configuration was previously proposed in a theoretical study of $\mathrm{MnPc} / \mathrm{F}_{16} \mathrm{CoPc}$ dimers, ${ }^{8}$ and the magnetic properties of $\mathrm{MnPc}$ and $\mathrm{CuPc}$ in this configuration were studied by $\mathrm{Wu}$ et al. ${ }^{9}$ Furthermore, this configuration was experimentally observed in a recent STM investigation of the growth of $\mathrm{FePc}$ multilayer films on $\mathrm{Au}(111)$, as the reciprocal arrangement of the molecules between the first and second FePc layers. ${ }^{27}$ In addition to these three models, we have studied a dimer with two MnPc lying on top of each other and rotated by $45^{\circ}$, a structure that was for example observed as the reciprocal geometry of two $\mathrm{CoPc}$ in the first two layers of films of CoPc grown on a $\mathrm{Pb}(111)$ substrate. ${ }^{17}$ After a geometry relaxation of all the structures, we find that $\alpha+$ is the one with lowest energy, as can be seen from Table 1 with the total energies, and it is the structure the rest of this study focuses on.

To get an estimation of the magnetic coupling between the $\mathrm{MnPc}$ molecules in the dimers, we computed the energy difference between the ferromagnetic (FM) and the antiferromagnetic (AFM) arrangements, $\Delta E_{\mathrm{FA}}$, for all the structures given in Table 1 . After geometry relaxation we obtain in all cases ferromagnetic coupling between the $\mathrm{Mn}$ 
Table 1. Total Energy Difference from the Ferromagnetic Ground State $(\alpha+)$ Geometry and Calculated Energy Difference between the Ferromagnetic and Antiferromagnetic Configurations $\left(\Delta E_{\mathrm{FA}}\right)$ for All the Dimers in the Gas Phase

\begin{tabular}{lcccl} 
& \multicolumn{4}{c}{ structures } \\
\cline { 2 - 5 } & $\alpha+$ & $\beta$ & top $45^{\circ}$ & $\alpha \times$ \\
$\Delta E(\mathrm{meV})$ & 0.0 & 168 & 240 & 322 \\
$\Delta E_{F A}(\mathrm{meV})$ & 257 & 184 & 289 & 25 \\
\hline
\end{tabular}

centers (corresponding to a positive value of $\Delta E_{\mathrm{FA}}$ ), but with different magnitudes in the various configurations. The top $45^{\circ}$ dimer has the strongest coupling but has a higher total energy. In the dimers, both the $\alpha+$ and the $\alpha \times$ actually have a ferromagnetic coupling according to our calculations unlike what is reported for the $\alpha$ polymorphs. ${ }^{11,12} \Delta E_{\mathrm{FA}}$ is higher in the $\alpha+$ and in the $\beta$ dimers than in the $\alpha \times$. We attribute this to the different mechanisms that govern the magnetic coupling between the two $\mathrm{Mn}$ atoms which depend on their relative positions. In fact, different tilt angles in the dimers, resulting from different shifts between the planes of the two molecules, are generally expected to affect the superexchange mechanism, since the relative orientation of the molecules influences the possible overlap between different molecular orbitals. In the $\alpha+$ dimer, each $\mathrm{Mn}$ atom is located precisely on top of a $N_{\text {iso }}$ of the other MnPc. From the side view of Figure 1B, interestingly, it is noticeable that the MnPcs in the dimer are indeed influenced by the presence of the opposite phthalocyanine, since they are not as flat as the single molecule in gas phase (Figure 1A). The two central Mn atoms are $3.20 \AA$ apart and slightly protruding from the plane of the molecule toward each other. For $\mathrm{MnPc}$ in gas phase, the computed bonding distances between the $\mathrm{Mn}$ and the $N_{\text {iso }}$ atoms amount to 1.97 and $1.96 \AA$ in the two orthogonal directions because of the $D_{2 h}$ symmetry of the molecule induced by Jahn-Teller distortions. ${ }^{23,28}$ When the dimer is formed, the $D_{2 h}$ symmetry is lifted. The distance between the $\mathrm{Mn}$ and the $N_{\text {iso }}$ that is facing the $\mathrm{Mn}$ of the other molecule is now $1.98 \AA$, slightly elongated with respect to the gas phase bond lengths, while the bonding distance with the three remaining $N_{\text {iso }}$ is $1.96 \AA$.

In the Pc films, the magnetic coupling between the metal atoms is generally explained by an intermolecular superexchange interaction that connects two successive Mn centers along the chains through the $\mathrm{N}$ atoms of the closest molecules. The interaction depends on the overlap of the $3 \mathrm{~d}$ orbitals of $\mathrm{Mn}$ with the $\pi$ orbitals centered on the $\mathrm{N}$ atoms in the organic shell. $^{12}$
As in the $\alpha$ and $\beta$ phases, the relevant orbitals extending between the two molecules are the out-of-plane $\mathrm{Mn} 3 \mathrm{~d}_{z}{ }^{2}$ and the $3 \mathrm{~d}_{\pi}$ (i.e., the $3 \mathrm{~d}_{x z}$ and $3 \mathrm{~d}_{y z}$ ) and the $N_{\text {iso }} 2 \mathrm{p}_{z}$. One should point out that these electrons actually participate in molecular orbitals that involve also other atoms of the $\operatorname{MnPc}(\mathrm{C}, \mathrm{N}$, and $\mathrm{Mn}$ ). Furthermore, more than one orbital combination may contribute to the superexchange interaction, generating a more complex process than the one usually described. In a simplified scheme, the ferromagnetic superexchange coupling in the $\beta$ phase is assumed to occur mainly via the overlapping of the Mn $3 \mathrm{~d}_{z}{ }^{2}$ with the $\mathrm{N}(\pi)$, while the antiferromagnetic coupling in the $\alpha$ phase via the overlapping of the $\mathrm{Mn}_{\pi}$ with the $\mathrm{N}(\pi)$ orbitals. In the ferromagnetic $\alpha+$ configuration, the atoms involved in the superexchange path form a loop that develops along the $\mathrm{Mn}$ centers and two of the $\mathrm{N}_{\text {iso }}$ atoms located on the opposite $\mathrm{MnPc}$, while in the $\beta$ configuration, shown in Figure $\mathrm{S} 1$, the superexchange path develops instead along the $\mathrm{Mn}$ atoms and the $\mathrm{N}_{\text {aza }}$ atoms. These four atoms in the $\alpha+$ configuration form a rectangular loop that is highlighted in Figure 2 for three of the different structures based on the $\alpha+$ dimer considered in this study and that will be discussed in the next paragraphs, namely $(\mathrm{MnPc})_{2}$ in gas phase (Figure $2 \mathrm{~A}$ ) and adsorbed on the Co substrate (Figure $2 \mathrm{~B}$ ), and $(\mathrm{MnPc})_{2}$ with a $\mathrm{Cl}$ axial ligand (Figure $2 \mathrm{C}$ ). The two $\mathrm{Mn}$ atoms in the loop are indicated as $\mathrm{Mn} 1$ and $\mathrm{Mn} 2$ and the $N_{\text {iso }}$ in front as N1 and $\mathrm{N} 2$, where the indices 1 and 2 identify the two molecules, 1 being the upper $\mathrm{MnPc}$, on which the axial ligands are adsorbed, and 2 being the index of the molecule directly adsorbed on $\mathrm{Co}(001)$. In the bare dimer in gas phase, the superexchange loop has a regular rectangular shape with intramolecular distances between the $\mathrm{Mn}$ atoms and the $N_{\text {iso }}$ of $1.98 \AA$ and intermolecular distance between the $\mathrm{Mn}$ atoms and the $N_{\text {iso }}$ of $2.74 \AA$. In this configuration, we obtain the highest ferromagnetic coupling, with a $\Delta E_{\mathrm{FA}}$ value of $257 \mathrm{meV}$. The atomic distances in the four-atom loop for all the configurations studied with the respective $\Delta E_{\mathrm{FA}}$ are reported in Table 2.

The next step was to simulate the deposition of the $\alpha+$ dimer on a $\mathrm{Co}(001)$ surface. In our previous works regarding the adsorption of $\mathrm{FePc}$ on $\mathrm{Co}(001)$, we found a ground state corresponding to the molecule adsorbed on a top site, that is, with the $\mathrm{Fe}$ atom on top of a Co atom and with the $\mathrm{Mn}-\mathrm{N}_{\text {iso }}$ main axes of the molecule oriented along the [100] crystallographic direction of the surface. ${ }^{16,26}$ The adsorption of a single $\mathrm{MnPc}$ on the same surface gives analogous outcomes for structures and adsorption sites as for the FePc, as reported in Table S1. Following these results, we have placed the whole $(\mathrm{MnPc})_{2}$ dimer on the same top Co site, and the

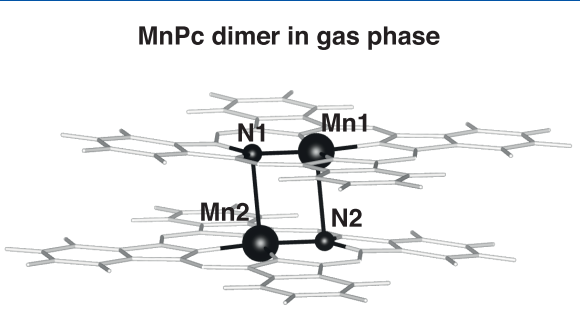

A

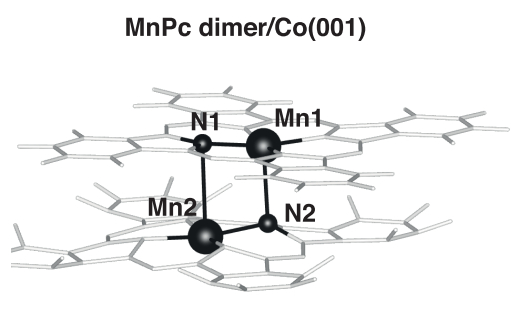

B

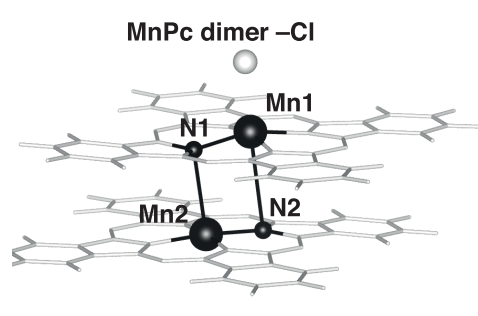

C

Figure 2. Four-atom loop along which the superexchange interaction $\mathrm{Mn}-\mathrm{N}-\mathrm{Mn}$ can take place between the two MnPc's in the dimer: the atoms involved in the loop are indicated as Mn1 and N1 on MnPc1, and Mn2 and N2 on MnPc2. (A) The loop in the gas phase, (B) in the chemisorbed dimer (where the surface located below the lower $\mathrm{MnPc}(\mathrm{MnPc} 2)$ is omitted for clarity), and $(\mathrm{C})$ in the dimer with a $\mathrm{Cl}$ axial ligand bonded to $\mathrm{Mn} 1$. 
Table 2. Energy Difference between the Ferromagnetic (FM) and Antiferromagnetic (AFM) Configurations $\left(\Delta E_{\mathrm{FA}}, \mathrm{meV}\right)$ and Interatomic Distances $(\AA)$ within the Superexchange Loop for All the Configurations Studied

\begin{tabular}{|c|c|c|c|c|c|c|}
\hline structure & $\Delta E_{\mathrm{FA}}$ & $\mathrm{Mn} 2-\mathrm{Mn} 1$ & $\mathrm{Mn} 1-\mathrm{N} 2$ & $\mathrm{~N} 2-\mathrm{Mn} 2$ & $\mathrm{Mn} 2-\mathrm{N} 1$ & $\mathrm{~N} 1-\mathrm{Mn} 1$ \\
\hline$(\mathrm{MnPc})_{2}$ & 257 & 3.20 & 2.74 & 1.98 & 2.74 & 1.97 \\
\hline$(\mathrm{MnPc})_{2} / \mathrm{Co}(001)$ & 15 & 3.32 & 2.46 & 2.03 & 2.94 & 1.98 \\
\hline $\mathrm{H}-(\mathrm{MnPc})_{2}$ & 203 & 3.26 & 2.83 & 1.97 & 2.70 & 1.97 \\
\hline $\mathrm{H}-(\mathrm{MnPc})_{2} / \mathrm{Co}(001)$ & -6 & 3.41 & 2.58 & 2.03 & 2.90 & 1.98 \\
\hline $\mathrm{Cl}-(\mathrm{MnPc})_{2}$ & 18 & 3.62 & 3.03 & 2.01 & 2.97 & 2.00 \\
\hline $\mathrm{Cl}-(\mathrm{MnPc})_{2} / \mathrm{Co}(001)$ & 15 & 3.71 & 3.03 & 2.01 & 2.98 & 2.00 \\
\hline
\end{tabular}

relaxed structure is displayed in Figure 1C. The chemisorption casts the dimer into a heterogeneous environment with one phthalocyanine $(\mathrm{MnPc} 2)$ in contact with the metal and the other one $(\mathrm{MnPcl})$ facing vacuum. Hence, the structural symmetry between the two molecules is lifted, with an impact on the overall geometry and properties of the dimer. A sideview inspection of the adsorbed dimer (Figure 1C) shows that $\mathrm{MnPc2}$ is subject to noticeable distortions of the planar symmetry, while $\mathrm{MnPcl}$ maintains a flatter geometry, akin to the gas phase molecule. The distance between the $\mathrm{Mn}$ atoms (Mn2 in the dimer) and the Co atom beneath is $2.43 \AA$ for the single molecule and $2.36 \AA$ for the adsorbed dimer, while the distance between the two $\mathrm{Mn}$ atoms for the adsorbed dimer is $3.32 \AA$, larger by $0.12 \AA$ than in the gas phase. These distances are similar to those obtained by means of a X-rays standing wave experiment of $\mathrm{MnPc}$ adsorbed on $\mathrm{Cu}(001)$ which gave an adsorption distance of $2.240 \pm 0.045 \AA$ for the $\mathrm{Mn}$ to the top layer of the surface. ${ }^{29}$ When the MnPc dimer is adsorbed on $\mathrm{Co}(001)$, we still obtain ferromagnetic coupling between the two molecules and between the dimer and the substrate (see Table 2). However, we find that the magnetic coupling between the two MnPc's is dramatically decreased, with $\Delta E_{\mathrm{FA}}$ reduced to $15 \mathrm{meV}$. In practice, we find that the deposition of the dimer on the Co substrate severely weakens the magnetic coupling between the molecules. This result may imply that in a magnetic nanodevice based on a chain or on a multilayer of $\mathrm{MnPc}$ the first layer would not be magnetically coupled to the remaining layers. However, the magnetic moments on the $\mathrm{Mn}$ centers are only very little affected by the adsorption, while for similar compounds, like for $\mathrm{CoPc}$ on $\mathrm{Pb}(111),{ }^{30}$ the first molecular layer acts as a spin insulating buffer. The quenching of the coupling is reflected by the distortion of the superexchange path presented in Figure 2. In fact, the chemical bonding formed between the Mn1 center and the Co atom beneath alters the bond lengths between the $\mathrm{N}$ and $\mathrm{Mn}$ atoms reported in Table 2 . In the slightly corrugated geometry of the chemisorbed MnPc2 (Figure 1) the N2 atom is lifted toward $\mathrm{Mn} 1$, resulting in a shorter $\mathrm{Mn} 1-\mathrm{N} 2$ distance (2.46 $⿱$ ) and, on the other side, inducing a longer $\mathrm{Mn} 2-\mathrm{N} 1$ distance $(2.93$ $\AA$ ). This can be seen from the slightly irregular loop in Figure 2B. As is also the case for the MnPc dimer with $\mathrm{H}$ and $\mathrm{Cl}$ ligands, the increase of the intermolecular $\mathrm{Mn}-\mathrm{N}$ bond length between the two molecules affects the overlap of the orbitals along the loop and is correlated to the quenching of the coupling.

Experimental and theoretical studies of the axial adsorption of small molecules on several 3d metal Pc's have demonstrated how the ligands can influence the magnetic moment and spin state of the metal macrocycles. ${ }^{2,31,32}$ Here we focus on the effect of atomic $\mathrm{H}$ and $\mathrm{Cl}$ axial ligands and on how the coupling is additionally affected by the deposition on $\mathrm{Co}(001)$. When a $\mathrm{H}$ atom is adsorbed on one side of $(\mathrm{MnPc})_{2}$, the overall geometric structure of the dimer is only marginally changed with respect to the bare dimer (Figure S2A). After geometry relaxation, the distance between $\mathrm{Mn} 1$ and $\mathrm{Mn} 2$ is $3.26 \AA$, which is $0.06 \AA$ larger than in the bare dimer, and the $\mathrm{Mn}-\mathrm{N}_{\text {iso }}$ distances are between 1.95 and $1.98 \AA$ : in $\mathrm{MnPc1}$, bonded to $\mathrm{H}$, the $\mathrm{Mn}-\mathrm{N}_{\text {iso }}$ distances are generally shorter by just about $0.01 \AA$. The two MnPc have ferromagnetic coupling with a $\Delta E_{\mathrm{FA}}$ of $200 \mathrm{meV}$, which is $20 \%$ lower than in the bare dimer. In comparison to the gas phase dimer, the sides of the superexchange loop are only weakly distorted, with the Mn2$\mathrm{N} 1$ distance stretched from 2.74 to $2.83 \AA$ and the Mn1-N2 distance shortened from 2.74 to $2.70 \AA$ (see Table 2). The deposition of the dimer on the $\mathrm{Co}(001)$ surface on the top site (Figure S3A) leads instead to considerable changes. We obtain a quenching of the magnetic coupling with a $\Delta E_{\mathrm{FA}}$ of $-6 \mathrm{meV}$, where the minus sign indicates an antiferromagnetic character. In this case, we find a larger distance between the $\mathrm{Mn}$ centers of $3.41 \AA$. The Mn1-N2 bond length is $2.90 \AA$ and the N1Mn1 bond length is $2.03 \AA$, thus stretching two sides of the superexchange loop with respect to the bare dimer in the gas phase.

When a $\mathrm{Cl}$ ligand is adsorbed on $\mathrm{MnPc1}$ (Figure S2B), Mn1 is pulled toward the $\mathrm{Cl}$ atom, which lies $2.33 \AA$ above it. The $\mathrm{Mn} 1-\mathrm{Mn} 2$ distance is increased in the presence of $\mathrm{Cl}$ to 3.62 $\AA$ in the gas phase and to $3.71 \AA$ when adsorbed on the Co substrate (Figure S3B); in addition, all the bond lengths between the atoms in the superexchange loop are increased as reported in Table 2. The calculations show that in this case the $\mathrm{Cl}$ ligand brings about a drastic reduction of the coupling: the calculated $\Delta E_{\mathrm{FA}}$ is $18 \mathrm{meV}$ in the gas phase and $15 \mathrm{meV}$ when the dimer is adsorbed on Co (see Table 2). The coupling is ferromagnetic in both cases.

The distance between the Mn1-Mn2 atoms reported in Table 2 is not a major factor in determining the strength of the magnetic interaction: for example, it only varies by $0.06 \AA$ between $(\mathrm{MnPc})_{2}$ and $\mathrm{H}-(\mathrm{MnPc})_{2}$, which have $\Delta E_{\mathrm{FA}}$ differing by $20 \%$ from each other, and again it varies by $0.06 \AA$ between $\mathrm{H}-(\mathrm{MnPc})_{2}$ and $(\mathrm{MnPc})_{2}$ adsorbed on $\mathrm{Co}$, where the coupling is reduced to $15 \mathrm{meV}$. Rather, the coupling depends mostly on a more complex interplay between the orbital overlaps along the superexchange path. Summarizing the results of Table 2 for all the systems studied, we can point out how the magnetic coupling is considerable for only two structures, namely, for the bare dimer and for the dimer with the $\mathrm{H}$ ligand in gas phase. In these cases, the bond lengths within the superexchange loop are very similar, with intramolecular $\mathrm{Mn}-\mathrm{N}$ bond lengths between 1.97 and $1.98 \AA$ and intermolecular $\mathrm{Mn}-\mathrm{N}$ bond lengths between 2.70 and $2.83 \AA$. When the bare $(\mathrm{MnPc})_{2}$ is adsorbed on $\mathrm{Co}(001), \Delta E_{\mathrm{FA}}$ goes down to 15 $\mathrm{meV}$ : here the superexchange loop is distorted on one side, with the bond length Mn1-N2 increased to $2.94 \AA$, which is $0.2 \AA$ more than in gas phase. When $\mathrm{H}-(\mathrm{MnPc})_{2}$ is adsorbed 
Table 3. Magnetic Moments Obtained for Bare $(\mathrm{MnPc})_{2}$ and with $\mathrm{H}$ and $\mathrm{Cl}$ Ligands in the Gas Phase and Adsorbed on $\mathrm{Co}(001)$; Moments on the Mn, N, and C Atoms as Well as on the Ligand Atoms $\mathrm{H}$ and $\mathrm{Cl}$

\begin{tabular}{|c|c|c|c|c|c|c|c|}
\hline \multirow[b]{3}{*}{ configuration } & \multicolumn{7}{|c|}{ magnetic moments $\left(\mu_{\mathrm{B}}\right)$} \\
\hline & \multicolumn{3}{|c|}{$\mathrm{MnPc} 2$} & \multicolumn{4}{|c|}{$\mathrm{MnPc1}$} \\
\hline & $\mathrm{Mn}$ & $\mathrm{C}$ & $\mathrm{N}$ & $\mathrm{Mn}$ & $\mathrm{C}$ & $\mathrm{N}$ & ligand \\
\hline \multicolumn{8}{|c|}{ monomer/dimer in gas phase } \\
\hline $\mathrm{MnPc}$ & 3.5 & -0.24 & -0.20 & & & & \\
\hline$(\mathrm{MnPc})_{2}$ & 3.62 & -0.24 & -0.22 & 3.62 & -0.24 & -0.22 & \\
\hline $\mathrm{H}-(\mathrm{MnPc})_{2}$ & 3.65 & -0.24 & -0.22 & 2.95 & -0.27 & -0.23 & -0.13 \\
\hline $\mathrm{Cl}-(\mathrm{MnPc})_{2}$ & 3.62 & -0.21 & -0.21 & 3.74 & $3 \cdot 10^{-3}$ & -0.17 & 0.13 \\
\hline \multicolumn{8}{|c|}{ monomer/dimer on $\mathrm{Co}(001)$} \\
\hline $\mathrm{MnPc} / \mathrm{Co}(001)$ & 3.41 & -0.33 & -0.09 & & & & \\
\hline$(\mathrm{MnPc})_{2} / \mathrm{Co}(001)$ & 3.46 & -0.34 & -0.07 & 3.69 & -0.24 & -0.22 & \\
\hline $\mathrm{H}-(\mathrm{MnPc})_{2} / \mathrm{Co}(001)$ & 3.47 & -0.34 & -0.09 & -2.80 & 0.22 & 0.20 & 0.11 \\
\hline $\mathrm{Cl}-(\mathrm{MnPc})_{2} / \mathrm{Co}(001)$ & 3.40 & 0.33 & -0.08 & 3.74 & 0.04 & -0.15 & 0.13 \\
\hline
\end{tabular}

on Co, we obtain a $\Delta E_{\mathrm{FA}}$ of $-6 \mathrm{meV}$, the $\mathrm{Mn} 2-\mathrm{N} 1$ bond length is increased to $2.90 \AA$, and the Mn1-N1 bond length is increased to $2.01 \AA$. Finally, the dimer with $\mathrm{Cl}$ ligand has a $\Delta E_{\mathrm{FA}}$ equal to $18 \mathrm{meV}$ in the gas phase and $15 \mathrm{meV}$ when adsorbed on the Co substrate. The $\mathrm{Cl}$ ligand in general induces an increase of both the intra- and intermolecular $\mathrm{Mn}-\mathrm{N}_{\text {iso }}$ bond lengths, both in gas phase and on the surface.

Table 3 shows the computed magnetic moments on the Mn, $\mathrm{C}$, and $\mathrm{N}$ atoms and on the ligands for the monomer and for all the examined configurations of the $\alpha+$ dimers.

In $(\mathrm{MnPc})_{2}$ in the gas phase, in each molecule, the major part of the magnetic moment resides on the Mn atom $\left(3.6 \mu_{\mathrm{B}}\right)$, while about $12 \%$ of this moment with opposite sign $\left(-0.46 \mu_{\mathrm{B}}\right)$ is distributed among the $\mathrm{N}$ atoms and the $\mathrm{C}$ atoms bonded to them in the organic ring, as shown by the computed isosurface magnetization density for $(\mathrm{MnPc})_{2}$ in Figure 3A. This distribution of the magnetic moments is very similar to that of the single molecule in gas phase with $3.5 \mu_{\mathrm{B}}$ on $\mathrm{Mn}$ and $-0.44 \mu_{\mathrm{B}}$ on the $\mathrm{N}$ and $\mathrm{C}$ atoms in the organic ring.

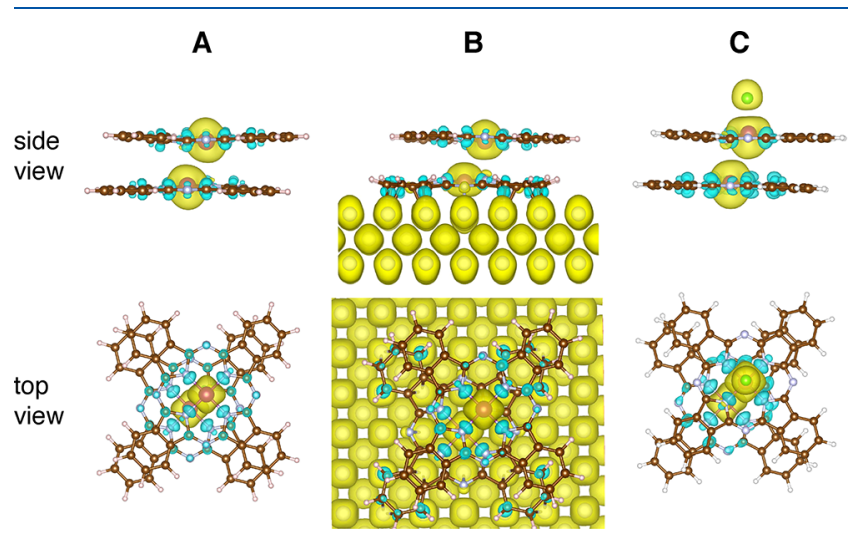

Figure 3. Side and top view of the isosurface magnetization density of (A) $(\mathrm{MnPc})_{2}$ in the gas phase, $(\mathrm{B})(\mathrm{MnPc})_{2}$ adsorbed on the $\mathrm{Co}(001)$ surface, and $(\mathrm{C})$ of $\mathrm{Cl}-(\mathrm{MnPc})_{2}$ in the gas phase. Yellow and light blue shapes indicate spin-up and -down densities, respectively.

When $\mathrm{MnPc}$ is adsorbed on $\mathrm{Co}(001)$, both as single molecule and as a dimer, we obtain a slightly smaller moment on the $\mathrm{Mn}$ atom directly in contact with the surface, varying between $3.41 \mu_{\mathrm{B}}$ for $\mathrm{MnPc}$ and 3.46 for $(\mathrm{MnPc})_{2}$, as can be seen in Table 3. At the same time, we see an increase in the moments of the $\mathrm{C}$ atoms in the benzene rings that bond to the Co surface by about $0.1 \mu_{\mathrm{B}}$ (see Figure $3 \mathrm{~B}$ ). On the other hand, the total moment of the $\mathrm{N}$ atoms decreases by about 1.1 $\mu_{\mathrm{B}}$, since here the moment in the ring is concentrated in the $\mathrm{N}_{\text {iso }}$ with negligible contributions from the $\mathrm{N}_{\mathrm{aza}}$. These results are similar to the variation of magnetic moments for $\mathrm{MnPc}$ adsorbed on $\mathrm{Cu}(001)$ computed with the same method by Alouani and co-workers, ${ }^{29}$ where between gas phase and adsorption on surface, a variation in the Mn moment from 3.46 to $3.34 \mu_{\mathrm{B}}$ was found, with a change of the total $\mathrm{C}$ moment from -0.15 to $-0.27 \mu_{\mathrm{B}}$ and a very small change of the total $\mathrm{N}$ moment from -0.15 to $-0.14 \mu_{\mathrm{B}}$. The small differences observed in the present work could be attributed not only to the different surfaces but also to the different adsorption sites considered in the two works, since this could affect the bonding and therefore the charge transfer of the $\mathrm{N}$ and $\mathrm{C}$ ligands to the substrate and finally their magnetic moments.

The axial ligands mainly modify the distribution of the moments in the central part of $\mathrm{MnPcl}$ where they are adsorbed. In $\mathrm{H}-(\mathrm{MnPc})_{2}$, the moment on $\mathrm{Mnl}$ is $2.95 \mu_{\mathrm{B}}$ with $-0.50 \mu_{\mathrm{B}}$ on the ring (about $17 \%$ of the moment on Mn1 but with opposite sign). The $\mathrm{H}$ ligand brings about a decrease of the moment on Mn1 accompanied by an increase of the moment on the $\mathrm{C}$ atoms. The $\mathrm{Cl}$ ligand, on the contrary, induces an increase of the magnetic moment on $\mathrm{Mn} 1$ to 3.74 $\mu_{\mathrm{B}}$. Now a moment of $-0.17 \mu_{\mathrm{B}}$ ( $5 \%$ of the moment on $\mathrm{Mn} 1$ ) is located on the ring and mostly concentrated on $\mathrm{N}_{\text {iso }}$, as can be seen from Figure 3C.

WhenH- $(\mathrm{MnPc})_{2}$ is adsorbed, the moment in $\mathrm{Mn} 1$ further decreases to $-2.80 \mu_{\mathrm{B}}$, and it goes to $0.44 \mu_{\mathrm{B}}$ on the ring. In Cl$(\mathrm{MnPc})_{2}$ the moment stays at $3.74 \mu_{\mathrm{B}}$ on $\mathrm{Mn} 1$, and it is lowered to -0.11 in the ring. The antiferromagnetic coupling observed in all these cases between the central metal and the organic ring was also been observed for several metal Pc's deposited on surfaces, ${ }^{29,33}$ while for some Pc-based 3D materials, it was found to be ferromagnetic, for example, in $\mathrm{FePc}(\mathrm{CN})_{2} \cdot 2 \mathrm{CHCl}_{3}$ crystals. $^{34}$

In addition, we have considered the magnetic interaction of the dimer to the surface, which takes place through MnPc2. We have obtained a ferromagnetic coupling of the $\mathrm{Mn} 2$ atom with the Co atoms as well as an antiferromagnetic coupling of the $\mathrm{C}$ atoms in $\mathrm{MnPc} 2$ to the surface. The same magnetic configuration was obtained for the single molecule and for the dimers with axial ligands. The bonding of the molecule to the surface occurs through the direct interaction of especially $\mathrm{Mn}$ and $\mathrm{C}$ atoms in the benzene rings to the underlying Co atoms, and this is coupled to variations in magnetic moment on both the $\mathrm{MnPc}$ and $\mathrm{Co}(001)$ surface. This results in a sort of 
footprint left by the molecule on the underlying surface, as can be seen in Figure 4, where the magnetic moment of the top Co

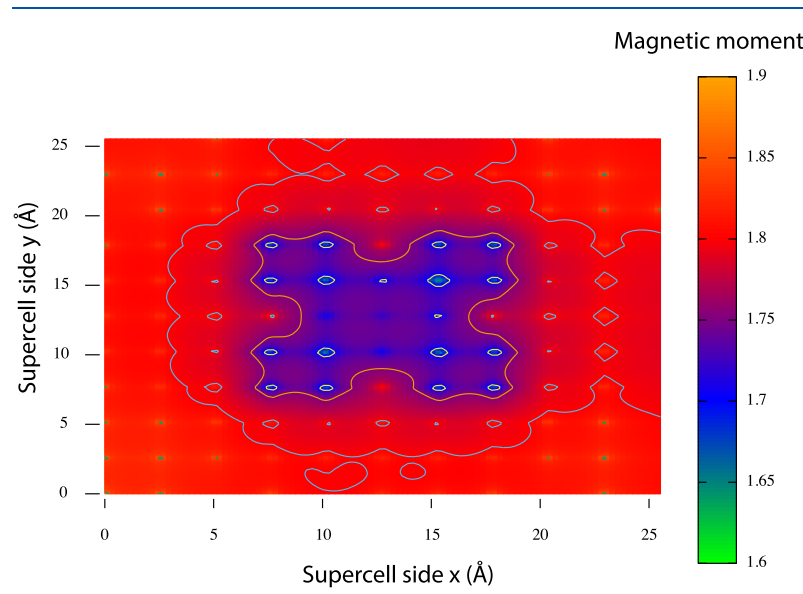

Figure 4. Magnetization density on the top Co surface layer below the adsorbed $(\mathrm{MnPc})_{2}$.

atoms on the surface are shown. A profile highlighting the position of the adsorbed molecule is also sketched. The magnetic moments of the Co atoms vary between about 1.6 and $1.9 \mu_{\mathrm{B}}$, and the strongest variations are given by those Co atoms lying below the $\mathrm{C}$ atoms in the benzene rings, suggesting the decisive role played by these atoms in the bonding of $\mathrm{MnPc}$ to the surface. We had previously found the same type of patterning for the adsorption of $\mathrm{FePc}$ on the same substrate. $^{16}$

We have finally examined the interplay between the chemical and geometrical configurations and the electronic structure of the $\mathrm{MnPc}$ molecules in the dimer and how this is related to the magnetic coupling. The partial density of states
(pDOS) of the single molecule and of the $\mathrm{MnPc}$ dimer in gas phase and on $\mathrm{Co}(001)$ are presented in Figure $5 \mathrm{~A}-\mathrm{C}$. In Figure $5 \mathrm{~A}$, the pDOS of the single molecule is illustrated, with the Mn $3 \mathrm{~d}$ orbitals, $3 \mathrm{~d}_{z^{2}}, 3 \mathrm{~d}_{\pi}, 3 \mathrm{~d}_{x y}$, and $3 \mathrm{~d}_{x^{2}-y^{2}}$, and the $N_{\text {iso }} 2 \mathrm{p}_{z}$. For the single $\mathrm{MnPc}$ in gas phase we obtain a ${ }^{4} \mathrm{E}_{\mathrm{g}}$ ground state where the $3 \mathrm{~d}_{z}{ }^{2}$ and the $3 \mathrm{~d}_{x y}$ are half occupied, and the $3 \mathrm{~d}_{\pi}$ contains three electrons (see Figure 5A), in agreement with previous DFT studies ${ }^{23,35}$ and experimental results. ${ }^{36,37}$ MnPc's maintain the ${ }^{4} \mathrm{E}_{\mathrm{g}}$ electronic configuration in the $\alpha+$ dimer and even when they are adsorbed on $\mathrm{Co}(001)$ and with the axial ligands (Figure 5B,C). Because the dimer is symmetric for the two molecules, only the pDOS for one of the two is shown in Figure 5B. First of all, one can notice how the $M_{\mathrm{n}} 3 \mathrm{~d}_{z^{2}}$ orbital in the dimer is split into several components of lower intensity (black lines in Figure 5). This is evident when comparing the two high-intensity $3 \mathrm{~d}_{z}{ }^{2}$ peaks of the single $\mathrm{MnPc}$ at -4.3 and $1.5 \mathrm{eV}$ in Figure 5A, with the several low-intensity $3 \mathrm{~d}_{z^{2}}$ peaks in the regions between -5.4 and $-3.8 \mathrm{eV}$ and between 0.8 and $2.6 \mathrm{eV}$ in the gas phase dimer in Figure 5B, and is a result of the interaction between the two molecules. Also, the $3 \mathrm{~d}_{\pi}$ states (gray curves) undergo some minor changes in energy position and especially in intensity, although they do not further split into several smaller components, giving a hint that the $3 \mathrm{~d} \pi$ states are less involved in the hybridization between the molecules. In all the diagrams, also the partial DOS of $N_{\text {iso }} 2 \mathrm{p}_{z}$ is plotted, for the specific $N_{\text {iso }}$ which is located opposite to a $\mathrm{Mn}$ atom of the other molecule; this pDOS is very weak compared to that of the Mn 3d electrons, and its intensity is therefore multiplied by a factor 4 . In $(\mathrm{MnPc})_{2}$ the $N_{\text {iso }} 2 \mathrm{p}_{z}$ pDOS overlaps the Mn 3d orbitals in several energy intervals, like for example between -5 and -4 $\mathrm{eV}$, at about 0.5 and $2 \mathrm{eV}$ in Figure 5B, suggesting hybridization with the Mn $3 \mathrm{~d}$ electrons. In this dimer as we
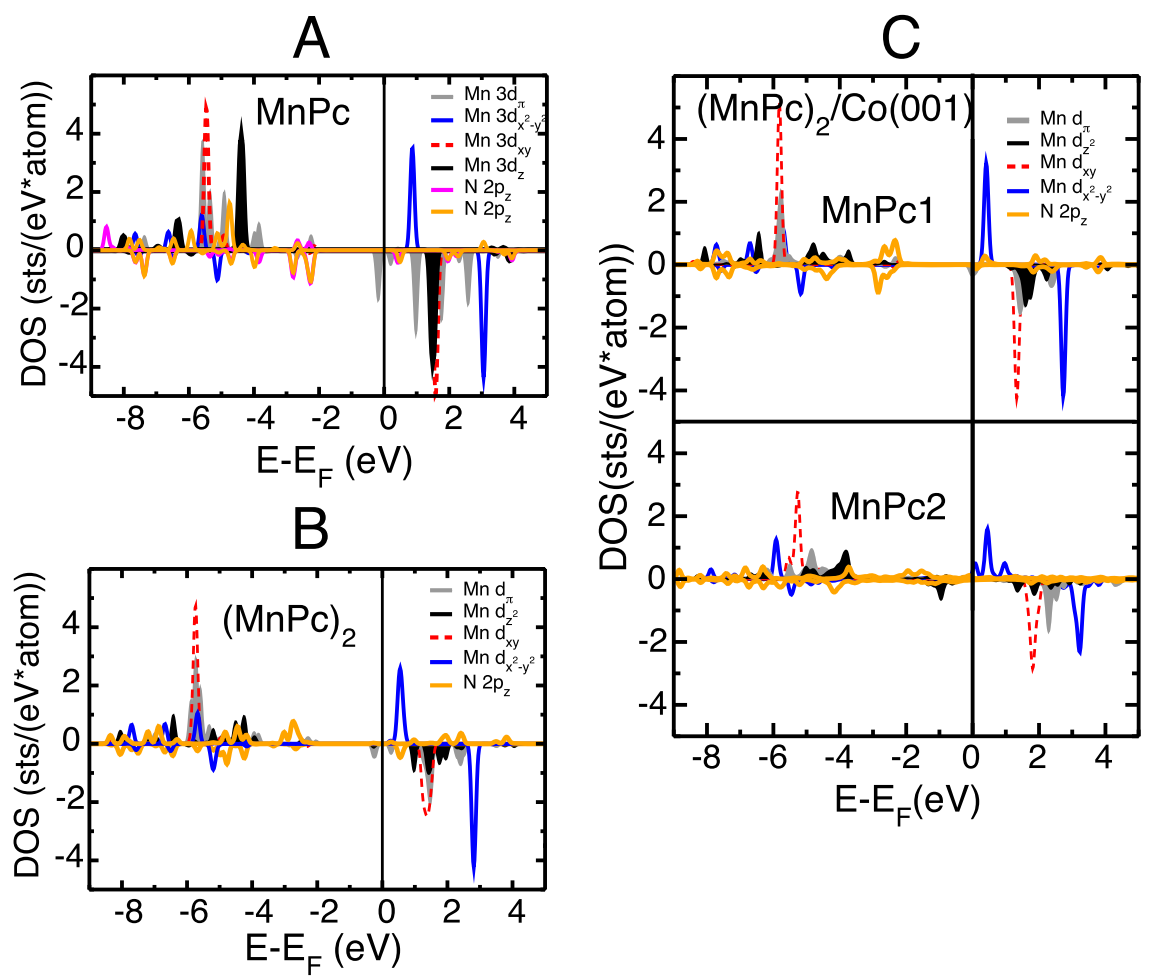

Figure 5. Partial DOS of $(\mathrm{A})$ a single $\mathrm{MnPc}$ in gas phase, $(\mathrm{B})$ of $(\mathrm{MnPc})_{2}$ in gas phase, and $(\mathrm{C})$ of $(\mathrm{MnPc})_{2}$ deposited on Co(001). 

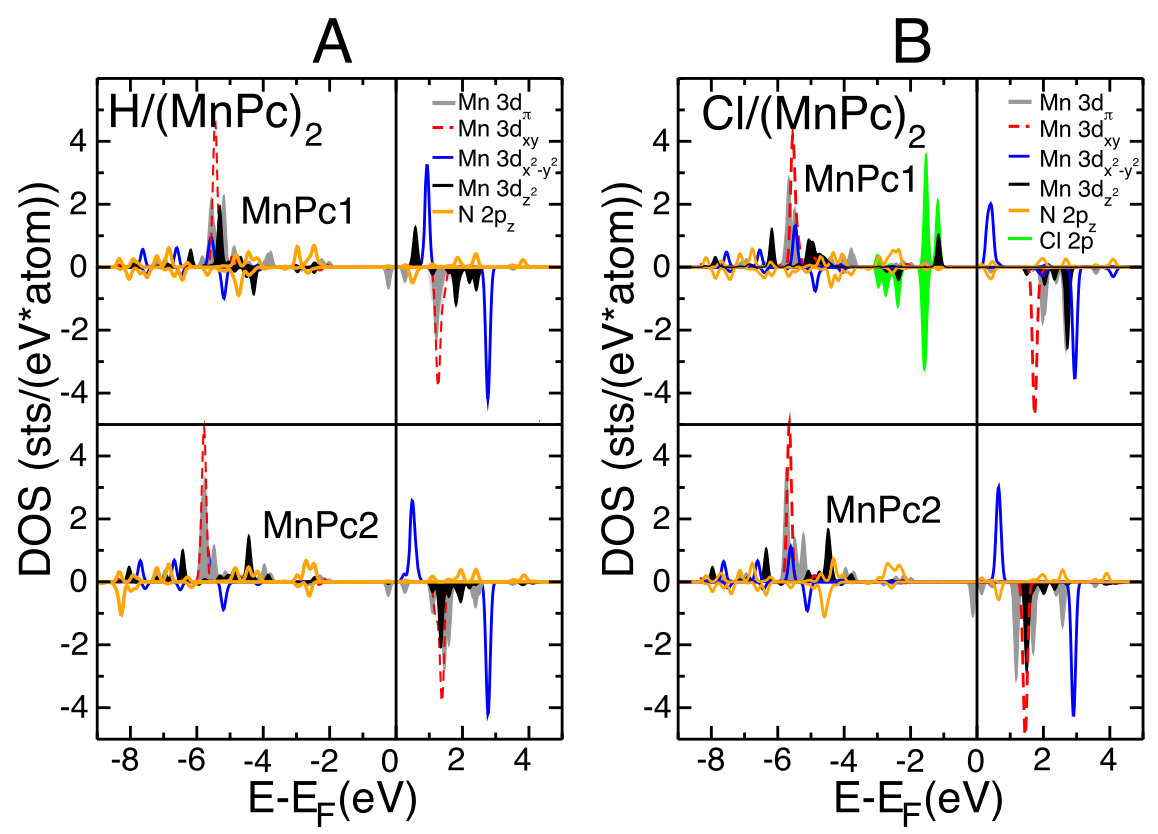

Figure 6. (A) Partial DOS of $\mathrm{Cl}-\mathrm{MnPc}$ in the gas phase and (B) $\mathrm{H}-(\mathrm{MnPc})_{2}$ in the gas phase.

have discussed before, the magnetic coupling with a $\Delta E_{\mathrm{FA}}$ of $256 \mathrm{meV}$ is strong compared to other dimer configurations.

When the MnPc dimer is adsorbed on $\mathrm{Co}(100)$, the lower molecule (MnPc2) is strongly bonded to the substrate. The $\mathrm{Mn}$ center on a top site bonds to the Co atom above through the out of plane $3 \mathrm{~d}_{z}{ }^{2}$ and $3 \mathrm{~d}_{\pi}$ electrons. In this configuration, the $\mathrm{MnPc}$ is ferromagnetically coupled to the Co surface, in analogy to $\mathrm{FePc}$ on $\mathrm{Co}(100)$, as we had reported in our previous studies ${ }^{16,26}$ and as was also found experimentally for example for the adsorbed systems $\mathrm{FePc}, \mathrm{CoPc}, \mathrm{NiPc}$, and $\mathrm{CuPc}$ on $\mathrm{Ag}(100), \mathrm{FePc}, \mathrm{CoPc}$, and $\mathrm{CuPc}$ on $\mathrm{Co}(001),{ }^{38}$ and $\mathrm{MnPc}$ on $\mathrm{Co}(001){ }^{3}$ The pDOS curves of MnPc2 in Figure 5C show that both the $M n 3 d_{z}{ }^{2}$ and $3 d_{\pi}$ electrons are strongly affected by the adsorption, with the formation of several peaks with reduced intensity. The involvement of these electrons into the bonding to the surface corresponds to a somewhat weakening of the bonding between $\mathrm{MnPc1}$ and $\mathrm{MnPc} 2$, which could also be deduced by the flatter geometrical structure of $\mathrm{MnPc1}$, and is also responsible for the drastic decrease of the magnetic coupling between the molecules with a $\Delta E_{\mathrm{FA}}$ of $15 \mathrm{meV}$.

Figure $6 \mathrm{~A}$ shows the pDOS of $(\mathrm{MnPc})_{2}$ with adsorbed $\mathrm{H}$. Although the ferromagnetic coupling is only reduced by $20 \%$ from the bare dimer, some differences in the DOS of $\mathrm{MnPc1}$ are evident. For example in the Mn1 $3 \mathrm{~d}_{z}{ }^{2}$ pDOS we observe a different splitting of the peaks and a new $3 \mathrm{~d}_{z}{ }^{2}$ feature at 0.57 $\mathrm{eV}$. However, the pDOS of the MnPc2 and the $N_{\text {iso }} 2 \mathrm{p}_{z}$ are mostly analogous to the ones of the bare dimer.

Figure $6 \mathrm{~B}$ reports the pDOS of the dimer with the $\mathrm{Cl}$ ligand. In this case, a significant weakening of the magnetic coupling between the Mn centers occurs, as when the dimer is adsorbed on the $\mathrm{Co}(001)$. The $\mathrm{Cl}$ is adsorbed on $\mathrm{MnPc1}$, forming chemical bonds between the $M_{\mathrm{n}} 3 \mathrm{~d}_{z}{ }^{2}$ and $3 \mathrm{~d}_{\pi}$ electrons to the $\mathrm{Cl} 2 \mathrm{p}$ (see Figure $6 \mathrm{~B}$ between -3 and $-1 \mathrm{eV}$ ). These new bonds weaken the interaction between the two MnPc's significantly, which are now at $3.71 \AA$ from each other. This effect is also visible in the pDOS of $\mathrm{MnPc2}$ of Figure 5C, which resembles very closely the one of the gas phase $\mathrm{MnPc}$, with distinct peaks for the $M_{\mathrm{n}} 3 \mathrm{~d}_{z^{2}}$ and $3 \mathrm{~d}_{\pi}$ electrons. On the other side, the pDOS of MnPcl is highly affected by the bonding to $\mathrm{Cl}$.

For all these gas phase and adsorbed systems, the pDOSs provide further evidence of the role of the $M_{\mathrm{n}} 3 \mathrm{~d}_{z}^{2}$ and $3 \mathrm{~d}_{\pi}$ in creating the superexchange ferromagnetic interaction between the two Mn moments, since in all cases when these orbitals are involved in other chemical bondings, the superexchange path is disturbed and the coupling gets affected.

\section{CONCLUSIONS}

To summarize, the analysis of the interplay between the reciprocal arrangement of the molecules and the magnetic interaction in the $\alpha+$ dimer confirms that the magnetic coupling between the two MnPc takes principally place via superexchange interaction, exploiting one of the two paths in a loop entangling the $\mathrm{Mn}$ centers and two isoindole $\mathrm{N}$ atoms. The path is conceptually analogous to the one pointed out for the $\alpha$ and $\beta$ bulk phase, although in the dimers only ferromagnetic coupling is obtained. In the dimer, it is possible to generate distortions in the atomic loop, for example, by chemisorption on a substrate or by adsorption of axial ligands. We have highlighted how the coupling between the molecules can be tuned by ligand adsorption, and how it can be influenced by the adsorption of the dimer on the FM Co(001). In addition, $\mathrm{MnPc}$ has a ferromagnetic coupling via the $\mathrm{Mn}$ atom to the underlying surface, but the $\mathrm{C}$ and $\mathrm{N}$ atoms have an antiferromagnetic coupling to the same.

\section{ASSOCIATED CONTENT}

\section{Supporting Information}

The Supporting Information is available free of charge at https://pubs.acs.org/doi/10.1021/acs.jpcc.0c08448.

Structures and total energies of $\mathrm{MnPc}$ dimers and structures and density of states of $\mathrm{MnPc}$ dimers with $\mathrm{H}$ and $\mathrm{Cl}$ ligands adsorbed on $\mathrm{Co}(001)$ (PDF) 


\section{AUTHOR INFORMATION}

Corresponding Author

Barbara Brena - Department of Physics and Astronomy, Uppsala University, 75120 Uppsala, Sweden; (1) orcid.org/ 0000-0003-0503-4691; Email: barbara.brena@ physics.uu.se

\section{Authors \\ Biplab Sanyal - Department of Physics and Astronomy, Uppsala University, 75120 Uppsala, Sweden \\ Heike C. Herper - Department of Physics and Astronomy, Uppsala University, 75120 Uppsala, Sweden}

Complete contact information is available at: https://pubs.acs.org/10.1021/acs.jpcc.0c08448

\section{Notes}

The authors declare no competing financial interest.

\section{ACKNOWLEDGMENTS}

The funding from the Swedish Research Council (VR) and Carl Tryggers Foundation and eSSENCE is acknowledged as well as the Swedish National Infrastructure for Computing (SNIC) for providing computing time on the Beskow cluster at PDC, Stockholm, and at the Tetralith Cluster at NSC, Linköping. B.S. acknowledges supercomputing allocation time for the project "DYNAMAT" from PRACE.

\section{REFERENCES}

(1) Schmaus, S.; Bagrets, A.; Nahas, Y.; Yamada, T. K.; Bork, A.; Bowen, M.; Beaurepaire, E.; Evers, F.; Wulfhekel, W. Giant Magnetoresistance Through a Single Molecule. Nat. Nanotechnol. 2011, 6, 185-189.

(2) Liu, L.; Yang, K.; Jiang, Y.; Song, B.; Xiao, W.; Li, L.; Zhou, H.; Wang, Y.; Du, S.; Ouyang, M.; et al. Structure-Dependent Exchange in the Organic Magnets $\mathrm{Cu}(\mathrm{II}) \mathrm{Pc}$ and $\mathrm{Mn}(\mathrm{II}) \mathrm{Pc}$. Sci. Rep. 2013, 3, 1210 EP.

(3) Djeghloul, F.; Ibrahim, F.; Cantoni, M.; Bowen, M.; Joly, L.; Boukari, S.; Ohresser, P.; Bertran, F.; Fèvre, P. L.; Thakur, P.; et al. Direct Observation of a Highly Spin-Polarized Organic Spinterface at Room Temperature. Sci. Rep. 2013, 3, 1272.

(4) Barraud, C.; Bouzehouane, K.; Deranlot, C.; Kim, D. J.; Rakshit, R.; Shi, S.; Arabski, J.; Bowen, M.; Beaurepaire, E.; Petroff, F.; et al. Phthalocyanine Based Molecular Spintronic Devices. Dalton Transactions 2016, 45, 16694-16699.

(5) Dediu, V.; Murgia, M.; Matacotta, F. C.; Taliani, C.; Barbanera, $S$. Room Temperature Spin Polarized Injection in Organic Semiconductor. Solid State Commun. 2002, 122, 181.

(6) Bagrets, A.; Schmaus, S.; Jaafar, A.; Kramczynski, D.; Yamada, T. K.; Alouani, M.; Wulfhekel, W.; Evers, F. Single Molecule Magnetoresistance with Combined Antiferromagnetic and Ferromagnetic Electrodes. Nano Lett. 2012, 12, 5131-5136.

(7) Banerjee, A.; Kundu, B.; Pal, A. J. Introducing Immobilized Metal Phthalocyanines as Spin-Injection and Detection Layers in Organic Spin-Valves: Spin-Tunneling and Spin-Transport Regimes. Org. Electron. 2017, 41, 173-178.

(8) Friedrich, R.; Lindner, S.; Hahn, T.; Loose, C.; Liebing, S.; Knupfer, M.; Kortus, J. Systematic Theoretical Investigation of the Phthalocyanine Based Dimer: $\mathrm{MnPc}^{\delta^{+}} / \mathrm{F}_{16} \mathrm{CoPc}^{\delta^{-}}$. Phys. Rev. B: Condens. Matter Mater. Phys. 2013, 87, 115423.

(9) Wu, W.; Kerridge, A.; Harker, A. H.; Fisher, A. J. StructureDependent Exchange in the Organic Magnets $\mathrm{Cu}(\mathrm{II}) \mathrm{Pc}$ and Mn(II)Pc. Phys. Rev. B: Condens. Matter Mater. Phys. 2008, 77, 184403.

(10) Serri, M.; Wu, W.; Fleet, L. R.; Harrison, N. M.; Hirjibehedin, C. F.; Kay, C. W.; Fisher, A. J.; Aeppli, G.; Heutz, S. High-
Temperature Antiferromagnetism in Molecular Semiconductor thin Films and Nanostructures. Nat. Commun. 2014, 5, 3079.

(11) Barraclough, C. G.; Martin, R. L.; Mitra, S.; Sherwood, R. C. Paramagnetic Anisotropy, Electronic Structure, and Ferromagnetism in Spin S = 32 Manganese(II) Phthalocyanine. J. Chem. Phys. 1970, $53,1638-1642$.

(12) Yamada, H.; Shimada, T.; Koma, A. Preparation and Magnetic Properties of Manganese(II) Phthalocyanine Thin Films. J. Chem. Phys. 1998, 108, 10256-10261.

(13) Sanvito, S. Molecular spintronics: The Rise of Spinterface Science. Nat. Phys. 2010, 6, 562-564.

(14) Cinchetti, M.; Dediu, V. A.; Hueso, L. E. Activating the Molecular Spinterface. Nat. Mater. 2017, 16, 507.

(15) Girovsky, J.; Nowakowski, J.; Ali, M. E.; Baljozovic, M.; Rossmann, H. R.; Nijs, T.; Aeby, E. A.; Nowakowska, S.; Siewert, D.; Srivastava, G.; et al. Long-Range Ferrimagnetic Order in a TwoDimensional Supramolecular Kondo Lattice. Nat. Commun. 2017, 8 , 15388.

(16) Klar, D.; Brena, B.; Herper, H. C.; Bhandary, S.; Weis, C.; Krumme, B.; Schmitz-Antoniak, C.; Sanyal, B.; Eriksson, O.; Wende, $\mathrm{H}$. Oxygen-Tuned Magnetic Coupling of Fe-Phthalocyanine Molecules to Ferromagnetic Co Films. Phys. Rev. B: Condens. Matter Mater. Phys. 2013, 88, 224424.

(17) Chen, X.; Fu, Y.-S.; Ji, S.-H.; Zhang, T.; Cheng, P.; Ma, X.-C.; Zou, X.-L.; Duan, W.-H.; Jia, J.-F.; Xue, Q.-K. Probing Superexchange Interaction in Molecular Magnets by Spin-Flip Spectroscopy and Microscopy. Phys. Rev. Lett. 2008, 101, 197208.

(18) Kresse, G.; Furthmüller, J. Efficiency of Ab-Initio Total Energy Calculations for Metals and Semiconductors Using a Plane-Wave Basis Set. Comput. Mater. Sci. 1996, 6, 15.

(19) Perdew, J.; Burke, S.; Ernzerhof, M. Generalized Gradient Approximation Made Simple. Phys. Rev. Lett. 1996, 77, 3865.

(20) Perdew, J.; Burke, S.; Ernzerhof, M. Comment on "Generalized Gradient Approximation Made Simple. Phys. Rev. Lett. 1997, 78, 1396.

(21) Kresse, G.; Joubert, J. From Ultrasoft Pseudopotentials to the Projector Augmented-Wave Method. Phys. Rev. B: Condens. Matter Mater. Phys. 1999, 59, 1758.

(22) Dudarev, S. L.; Botton, G. A.; Savrasov, S. Y.; Humphreys, C. J.; Sutton, A. P. Electron-Energy-Loss Spectra and the Structural Stability of Nickel Oxide:An LSDA+U Study. Phys. Rev. B: Condens. Matter Mater. Phys. 1998, 57, 1505.

(23) Brumboiu, I. E.; Haldar, S.; Lüder, J.; Eriksson, O.; Herper, H.; Brena, B.; Sanyal, B. Influence of Electron Correlation on the Electronic Structure and Magnetism of Transition-Metal Phthalocyanines. J. Chem. Theory Comput. 2016, 12, 1772-1785.

(24) Grimme, S. Semiempirical GGA-type Density Functional Constructed with a Long-Range Dispersion Correction. J. Comput. Chem. 2006, 27, 1787.

(25) Grimme, S.; Antony, J.; Ehrlich, S.; Krieg, H. A consistent and Accurate ab Initio Parametrization of Density Functional Dispersion Correction (DFT-D) for the 94 Elements H-Pu. J. Chem. Phys. 2010, 132, 154104.

(26) Herper, H. C.; Bhandary, S.; Eriksson, O.; Sanyal, B.; Brena, B. $\mathrm{Fe}$ Phthalocyanine on $\mathrm{Co}(001)$ : Influence of Surface Oxidation on Structural and Electronic Properties. Phys. Rev. B: Condens. Matter Mater. Phys. 2014, 89, No. 085411.

(27) Bartolomé, F.; Bunǔau, O.; García, L. M.; Natoli, C. R.; Piantek, M.; Pascual, J. I.; Schuller, I. K.; Gredig, T.; Wilhelm, F.; Rogalev, A.; et al. Molecular Tilting and Columnar Stacking of $\mathrm{Fe}$ Phthalocyanine Thin Films on $\mathrm{Au}(111)$. J. Appl. Phys. 2015, 117, 17A135.

(28) Andjelkovic, L.; Stepanovic, S.; Vlahovic, F.; Zlatar, M.; Gruden, M. Resolving the Origin of the Multimode Jahn-Teller Effect in Metallophthalocyanines. Phys. Chem. Chem. Phys. 2016, 18, 29122.

(29) Javaid, S.; Lebegue, S.; Detlefs, B.; Ibrahim, F.; Djeghloul, F.; Bowen, M.; Boukari, S.; Miyamachi, T.; Arabski, J.; Spor, D.; et al. Chemisorption of Manganese Phthalocyanine on $\mathrm{Cu}(001)$ Surface 
Promoted by van der Waals Interactions. Phys. Rev. B: Condens. Matter Mater. Phys. 2013, 87, 155418.

(30) Wang, Y.; Zheng, X.; Yang, J. Kondo Screening and Spin Excitation in Few Layer CoPc Molecular Assembly Stacking on $\mathrm{Pb}(111)$ Surface: a DFT+HEOM Study. J. Chem. Phys. 2016, 145, 154301 .

(31) Isvoranu, C.; Knudsen, J.; Ataman, E.; Schulte, K.; Wang, B.; Bocquet, M.-L.; Andersen, J. N.; Schnadt, J. Reversible Change of the Spin State in a Manganese Phthalocyanine by Coordination of $\mathrm{CO}$ Molecule. J. Chem. Phys. 2011, 134, 114711.

(32) Stróżecka, A.; Soriano, M.; Pascual, J. I.; Palacios, J. J. Reversible Change of the Spin State in a Manganese Phthalocyanine by Coordination of CO Molecule. Phys. Rev. Lett. 2012, 109, 147202.

(33) Mugarza, A.; Robles, R.; Krull, C.; Korytár, R.; Lorente, N.; Gambardella, P. Electronic and Magnetic Properties of MoleculeMetal Interfaces: Transition-Metal Phthalocyanines Adsorbed on Ag(100). Phys. Rev. B: Condens. Matter Mater. Phys. 2012, 85, 155437.

(34) Sánchez de Armas, R.; Calzado, C. J. Evaluation of the Giant Ferromagnetic $\pi-\mathrm{d}$ Interaction in Iron-Phthalocyanine Molecule. J. Phys. Chem. A 2018, 122, 1678-1690.

(35) Liao, M.-S.; Watts, J. D.; Huang, M.-J. DFT Study of Unligated and Ligated ManganeseII Porphyrins and Phthalocyanines. Inorg. Chem. 2005, 44, 1941-1949.

(36) Kroll, T.; Kraus, R.; Schönfelder, R.; Aristov, V. Y.; Molodtsova, O.; Hoffmann, P.; Knupfer, M. Transition Metal Phthalocyanines: Insight into the Electronic Structure From Soft X-Ray Spectroscopy. J. Chem. Phys. 2012, 137, No. 054306.

(37) Williamson, B. E.; VanCott, T. C.; Boyle, M. E.; Misener, G. C.; Stillman, M. J.; Schatz, P. N. Determination of the Ground State of Manganese Phthalocyanine in an Argon Matrix Using Magnetic Circular Dichroism and Absorption Spectroscopy. J. Am. Chem. Soc. 1992, 114, 2412-2419.

(38) Lach, S.; Altenhof, A.; Tarafder, K.; Schmitt, F.; Ali, M. E.; Vogel, M.; Sauther, J.; Oppeneer, P. M.; Ziegler, C. Metal-Organic Hybrid Interface States of A Ferromagnet/Organic Semiconductor Hybrid Junction as Basis For Engineering Spin Injection in Organic Spintronics. Adv. Funct. Mater. 2012, 22, 989-997. 